\title{
Bovine neonatal pancytopenia in calves in Poland
}

\author{
J.M. Jaśkowski' ${ }^{1}$ M.P. Polak ${ }^{2}$, A. Marszałek ${ }^{3}$, K. Nowak ${ }^{4}$, \\ H. Frąckowiak ${ }^{5}$, J. Olechnowicz ${ }^{1}$
}

${ }^{1}$ Department of Veterinary Medicine, Faculty of Animal Breeding and Biology,

Poznań University of Life Sciences, Wojska Polskiego 20, 60-625 Poznań, Poland

2 Department of Virology, National Veterinary Research Institute, Partyzantów 57, 24-100 Puławy, Poland

${ }^{3}$ Department of Clinical Pathomorphology, Poznań University of Medical Sciences, Department of Clinical

Pathomorphology and Collegium Medicum in Bydgoszcz, Nicolaus Copernicus University in Toruń,

Ludwik Rydgier Collegium Medicum in Bydgoszcz, Jagiellonska 13-15, 85-067 Bydgoszcz, Poland

${ }^{4}$ Department of Physiology and Biochemistry, Faculty of Animal Breeding and Biology,

Poznań University of Life Sciences, Wołyńska 35, Poznan, Poland

${ }^{5}$ Department of Animal Anatomy, Poznań University of Life Sciences,

Wojska Polskiego 71c, 60-625 Poznan, Poland

\begin{abstract}
Bovine neonatal pancytopenia (BNP), a newly emerged syndrome of discussed etiology in calves, has been diagnosed since 2006. Here we describe first cases of BNP in Poland. Between September 2008 and April 2011, 62 cases of BNP were diagnosed in dairy calves. Bleeding skin lesions were mostly pronounced in summer and early autumn. Severe thrombocytopenia was observed in all sick animals. All calves came from herds vaccinated against BVDV infection with PregSure BVD vaccine (Pfizer). Substitution of colostrum from dams of BNP positive calves with colostrum from dams from herds free of BNP was the only effective measure to avoid new cases in affected herds.
\end{abstract}

Key words: bovine neonatal pancytopenia, bleeding, diathesis, thrombocytopenia, ecchymosis

\section{Introduction}

Bovine neonatal pancytopenia (BNP) is a newly emerged calf syndrome of unknown cause, found in Europe since 2006 (Bell et al. 2009, Doll 2012). While morbidity is low, the case fatality rate is very high. Typical symptoms of cutaneous bleeding, petechiae and/or melaena are accompanied by severe leucopenia and thrombocytopenia. Animals die shortly after the clinical onset (Pardon et al. 2010). The aim of the study was to describe cases of BNP diagnosed in years 2008-2011 in Poland.

\section{Materials and Methods}

In herds affected with BNP newborn calves were separated from dams on the $1^{\text {st }}$ day of life. They were fed mother's colostrum 3x/d within $24 \mathrm{~h}$ after birth and then until the $10^{\text {th }}$ day, regular milk. Laboratory diagnosis included: CBC; blood chemistry; RT-PCR of blood serum for genetic material of BVDV type 1 and 2, BTV; PCR for PCV2. 


\section{Results}

Since September 2008, 62 cases of BNP from 13 herds were recorded in Poland. Affected animals were of various breeds and both genders. All BNP positive calves came from herds vaccinated against BVDV infection with PregSure BVD vaccine (Pfizer). The mothers were vaccinated in 2006-2008 once a year and in 2009-2010 twice a year. BNP cases were observed in progeny of heifers and primiparous cows. First clinical signs occured 9-30 days after birth (av. 14 d) mostly in the strongest animals. Skin bleeding was observed above eyes, behind ears, on the neck and along the back line, mainly in calves born in summer and early autumn. Faeces were usually solid and dark-brown. Slight pyrexia was observed in 2 calves. It usually took 4-6 hours from the appearance of skin lesions to death.

Necropsy was performed on 6 calves. General physical condition was good. Smudges of blood were visible on the head, neck, back and limbs. Pale mucous membranes, livid rim around the mandibular incisors and extravasations ventraly on the tongue were regarded as pathognomonic for BNP (72\% of cases) and immediately preceded skin bleeding and animal's death. Other lesions included: multiple subcutaneous extravasations on the neck and chest, petechiae on skeletal muscles, blood and watery fluid in visceral cavities, lymphadenopatia, splenomegalia with hemorrhages, enlargement, gray coloration of the intestine with liquid, almost black content, normal-sized, dark brown kidneys, marbled lungs with visible lobule structure, unclotted blood with white foam on cut sections of lungs, heart with congested coronary arteries and ventricles filled with unclotted, watery blood.

Average CBC values were: erythrocytes 4.43 x $10^{12} / \mathrm{L}$ (4.9-10.9), leukocytes 1.15 x 109/L (2.6-14.6), lymphocytes $0.39 \times 10^{9} / \mathrm{L}(1.0-6.4)$ and platelets 20.2 x 109/L (190-940). Hb, PCV, MID, MCH, MCHC and $\mathrm{MCV}$ all were at the lowest reference level. APTT, PT, fibrinogen and prothrombin index were 74.7, 21.9 s, $3.15 \mathrm{~g} / \mathrm{L}$ and 50.6, respectively.

Analysis of peripheral WBC counts in blood smears showed that granulocytes, monocytes, segmented granulocytes and myeloblasts had values within reference ranges.

Laboratory testing for BVDV type 1, 2 and BTV by PCR gave negative results in all samples. PCV2 genetic material was found in one calf from a herd kept together with pigs.

\section{Discussion}

First cases of BNP were recorded in Poland in 2007 and the majority in 2009-2011 (Jaśkowski et al. 2010). Higher temperature in spring and summer seems to enhance the bleeding symptoms, although also in calves lacking such signs, thrombocytopenia was very advanced. Generally, there was no correlation between pancytopenia and skin bleeding. Livid rim around the mandibular incisors and extravasations ventral on the tongue were most commonly observed lesions in BNP affected animals. Every herd with a history of BNP used a killed vaccine against BVDV infection withdrawn from the market in June 2010. Very recent studies indicate that colostrum and sera of mothers of BNP affected calves produce alloantibodies targeting antigens on the blood cells of their progeny and indeed in our experience substitution of that colostrum with first milk from healthy cows is the only effective way to avoid BNP in newborn calves (Bridger et al. 2011, Friedrich et al. 2011).

\section{References}

Bell CR, Scott PR, Sargison ND, Wilson DJ, Morrison L, Howie F, Willoughby K, Penny CD (2010) Idiopathic bovine neonatal pancytopenia in a Scottish beef herd. Vet Record 167: 938-940.

Bridger PS, Bauerfeind R, Wenzel L, Bauer N, Menge C, Thiel HJ, Reinacher M, Doll K (2011) Detection of colostrum-derived alloantibodies in calves with bovine neonatal pancytopenia. Vet Immunol Immunopathol 141: $1-10$

Doll K (2012) Bovine neonatal pancytopenia. XXVII World Buiatrics Congress 2012, Lisbon Portugal, 3-8 June, Proceedings, pp 71-76.

Friedrich A, Büttner M, Rademacher G, Klee W, Weber BK, Müller M, Carlin A, Assad A, Hafner-Marx A, Sauter-Louis CM (2011) Ingestion of colostrum from specific cows induces Bovine Neonatal Pancytopenia (BNP) in some calves. BMC Vet Res 7: 10.

Jaśkowski JM, Bukowska D, Urbaniak K, Bronicki M (2010) Severe bovine pancytopenia. Lecznica Dużych Zwierząt 4: 6-10.

Pardon B, Steukers L, Dierick J, Ducatelle R, Saey V, Maes S, Vercauteren G, De Clercq K, Callens J, De Bleecker K, Deprez P (2010) Haemorrhagic diathesis in neonatal calves: an emerging syndrome in Europe. Transbound Emerg Dis 57: 135-146. 